\title{
A prevalência de lombalgia em universitários: revisão de literatura
}

\author{
The prevalence of low back pain in college students: revisión de literatura \\ Prevalencia del dolor lumbar en estudiantes universitarios: literature review
}

Recebido: 02/10/2021 | Revisado: 10/10/2021 | Aceito: 13/10/2021 | Publicado: 16/10/2021

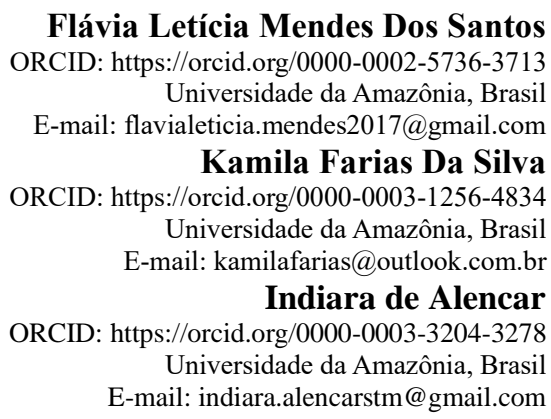

\begin{abstract}
Resumo
A lombalgia é classificada como um transtorno músculo esquelético muito comum sendo uma das principais causas que geram incapacidades pois envolve vários fatores de risco desencadeante, entre eles podemos citar hábitos posturais, fatores psicológicos, sedentarismo, longos períodos de tempo sentados e a falta de exercícios físicosNos universitários é possível encontrar muitos desses fatores por conta da rotina assídua de estudos, que acabam por impactar na qualidade de vida dos mesmo. Esse estudo trata-se de uma revisão de literatura com o objetivo de investigar a prevalência de lombalgia em universitários. Foram inclusos artigos escritos em idiomas nacionais e internacionais que tenham sido publicados entre os anos de 2012 à 2021 nas bases de dados eletrônicas Google Acadêmico, MedLine, PubMed, sciELO. Foi possível observar que houve alta prevalência de lombalgia em universitários, a maioria dos artigos expostos apresentaram em comum o acometimento de mais da metade dos participantes com queixas de lombalgia, sendo o gênero feminino mais afetado.
\end{abstract}

Palavras-chave: Prevalência; Lombalgia; Universitários.

\begin{abstract}
Low back pain is classified as a very common musculoskeletal disorder, being one of the main causes that generate disabilities as it involves several triggering risk factors, among them we can mention postural habits, psychological factors, sedentary lifestyle, long periods of sitting and lack of physical exercise. In university students it is possible to find many of these factors due to the assiduous study routine, which end up impacting their quality of life. This study is a literature review aiming to investigate the prevalence of low back pain in college students. Articles written in national and international languages that have been published between the years 2012 to 2021 in the electronic databases Google Academic, MedLine, PubMed, sciELO were included. It was possible to observe that there was a high prevalence of low back pain in college students, most of the articles exposed had in common the involvement of more than half of the participants with complaints of low back pain, with the female gender being more affected.
\end{abstract}

Keywords: Prevalence; Backache; College students.

\section{Resumen}

La lumbalgia se clasifica como un trastorno musculoesquelético muy común siendo una de las principales causas que generan discapacidades ya que involucra varios factores de riesgo desencadenantes, entre los que se pueden mencionar hábitos posturales, factores psicológicos, sedentarismo, largos períodos de estar sentado y carencia. de ejercicio físico. En los estudiantes universitarios es posible encontrar muchos de estos factores debido a la asidua rutina de estudio, que terminan impactando en su calidad de vida. Este estudio es una revisión de la literatura que tiene como objetivo investigar la prevalencia del dolor lumbar en estudiantes universitarios. Se incluyeron artículos escritos en idiomas nacionales e internacionales que han sido publicados entre los años 2012 a 2021 en las bases de datos electrónicas Google Academic, MedLine, PubMed, sciELO. Se pudo observar que existía una alta prevalencia de lumbalgia en estudiantes universitarios, la mayoría de los artículos expuestos tenían en común la participación de más de la mitad de los participantes con quejas de lumbalgia, siendo más afectado el género femenino.

Palabras clave: Predominio; Dolor de espalda; Estudiantes universitarios. 


\section{Introdução}

A dor lombar é definida como desconforto na região inferior da coluna vertebral, é um distúrbio musculoesquelético e pode se apresentar por uma combinação de fatores mecânicos, circulatórios, hormonais, psicossociais (Carvalho et al., 2017). Tipos de ocupação, alterações comportamentais, sedentarismo, sobrepeso (Bréder, 2018). Estilo de vida, e a permanência prolongada em certas posições (Da Silva Martins \& Cassiano Longen, 2017). Por ser a região do corpo que apresenta alta mobilidade a lombar está propensa a maior ocorrência de distúrbios que estão relacionados a inatividade física, apresentando características ligadas ao surgimento de dores que afetam devidamente essa extensão, prejudicando a execução dos movimentos e influenciando uma cascata de eventos que levam danos até nas características fisiológicas, pelas modificações mecânicas decorrentes a estas desordens (Miranda, 2007).

Atualmente vem se tornando-se um problema de saúde pública por intervir nas relações sociais, econômicas, profissionais e culturais, em que 70\% dos indivíduos em uma certa época de sua vida serão afetados (Barros et al., 2011). A lombalgia pode ser classificada dependendo do tempo e duração dos sintomas apresentados, sendo considerado aguda quando ocorre em períodos curtos e os sintomas perduram entre seis e doze semanas, e a crônica sucede por um tempo maior do que três meses (Ribeiro et al., 2018).

A fase de graduação é geralmente marcada pelo acréscimo de estresse e mudanças no estilo de vida (Serlachius et al., 2007). Nos acadêmicos, alterações estruturais e biomecânicas podem ocorrer por conta de posturas inadequadas na sala de aula, na maneira que transportam seus materiais e pela necessidade de permanecerem sentados por longos períodos (Gomes-Neto et al., 2016). A qualidade de vida dos universitários pode ser afetada diretamente pela dor lombar e por consequência diminuir o seu desempenho, sendo o ano do curso favorecido para o surgimento de complicações a coluna lombar (Morais et al., 2018). Evidências mostram que de acordo com o avanço do curso, os estudantes se tornam mais sedentários (Santos et al., 2014). Estudos confirmam que independente do grau acadêmico é grande a incidência para manifestação de lombalgia (Cervantes-Soto et al., 2019).

A perda da força e da flexibilidade muscular são aspectos que também estão relacionados a dor e sintomas frequentes nessa região, quando o indivíduo apresenta encurtamento dos músculos, assim como fraqueza muscular (Da Silva Martins \& Cassiano Longen, 2017). As alterações musculares ocasionadas por esse distúrbio possuem grande importância, uma vez que os músculos desempenham papel fundamental na estabilização da coluna lombar (Ebenbicher et al., 2001). O alongamento das cadeias musculares e articulares mostra eficácia na redução da dor e da incapacidade funcional, além de aumentar a flexibilidade global restabelecendo o equilíbrio dos músculos e articulações da região lombo pélvica, atuando como prevenção para lombalgia (Leite et al., 2015).

A lombalgia pode ser evitada ou reduzida com a execução de atividade física praticada constantemente, responsável por controlar a postura mantendo-a em posição ereta, com estabilização Intersegmentar, sendo requisitos importantes para a realização de atividades exercidas no cotidiano (Santos, 2011). Sendo a postura definida pelo equilíbrio estabelecido entre músculos, articulações e ossos. Tem como função a estabilização espacial do corpo humano e proteção conforme recebe estímulos do ambiente. Portanto a postura inadequada está relacionada à falta equilibrio com as estruturas de sustentação sendo a coluna vertebral a base principal, que atua como o eixo do corpo (Braccialli \& Villarta, 2000). Tem como objetivo investigar a prevalência de lombalgia em universitários.

\section{Metodologia}

Este estudo trata-se de uma revisão narrativa sendo está uma forma não sistematizada de revisar a literatura. Sempre buscando artigos mais recente a respeito de um determinado assunto, com um processo mais simplificado de rever a literatura. (Casarin et al, 2020). Sendo assim o nosso direcionamento para a questão problema é: "A Prevalência de lombalgia em universitários". Tendo como objetivo levantar o número de universitários afetados pela lombalgia. As bases de dados eletrônicas utilizadas foram Google Acadêmico, MEDline, PubMed, sciELO usando palavras-chave: Prevalência; Lombalgia; Universitários. 
Para o desenvolvimento os critérios de inclusão estabelecidos foram todos os artigos listados escritos em idiomas nacionais e internacionais que reportavam dados sobre dor lombar em universitários de (18 aos 30 anos) e que tenham sido publicados entre os anos de 2012 à 2021, artigos completos disponíveis integralmente nas bases de dados e relacionados com a relevância do tema. Os critérios de exclusão foram artigos que não fossem específicos na abordagem sobre a queixa dor lombar em universitários, artigos duplicados, incompletos, resumos, resenhas, debates, artigos publicados a mais de 10 anos.

A pesquisa iniciou-se no mês de julho de 2021 tendo término no fim do mês de setembro. As variáveis de interesse (primeiro autor, ano de publicação, tipo de estudo, ferramenta de coleta, tamanho amostral, população, idade, prevalência, lombalgia, universitário) foram anotadas pelo autor.

\section{Resultados e Discussão}

No Quadro 1 mostra-se 13 estudos, voltados para análise, onde tais estudos foram realizados em ambos os gêneros, todos submetidos a dores lombar em universitários.

Quadro 1 - Resultado dos estudos publicados nos anos de 2012 a 2021.

\begin{tabular}{|c|c|c|c|c|c|}
\hline Título & Autor e Ano & Amostra & Gênero & Objetivo & Resultados \\
\hline $\begin{array}{l}\text { Características principais da } \\
\text { dor lombar e deficiência em } \\
\text { adultos na } \\
\text { universitária: um } \\
\text { piloto. }\end{array}$ & $\begin{array}{l}\text { HANDRAKI } \\
\text { S, J. P, et al, } \\
2012 .\end{array}$ & $\begin{array}{l}84 \\
\text { universitário } \\
\text { s. Entre } 18 \text { e } \\
30 \text { anos }\end{array}$ & $\begin{array}{l}59 \% \\
\text { femininos } \\
34 \% \\
\text { masculinos. }\end{array}$ & $\begin{array}{l}\text { Identificar quais fatores } \\
\text { comumente associados à } \\
\text { lombalgia e e } \\
\text { incapacidade diferem } \\
\text { entre os universitários } \\
\text { com lombalgia e sem } \\
\text { lombalgia ou com } \\
\text { lombalgia mínima. }\end{array}$ & $\begin{array}{l}\text { Concluiu que indivíduos em } \\
\text { idade } \\
\text { universitária com maior } \\
\text { fortalecimento nas costas e } \\
\text { níveis mais altos de } \\
\text { atividade física tem menos } \\
\text { prevalencia de lombalgia e } \\
\text { deficiência. }\end{array}$ \\
\hline $\begin{array}{l}\text { Prevalência de dor lombar } \\
\text { em universitários da saúde e } \\
\text { sua relação com estilo de } \\
\text { vida e nível de atividade } \\
\text { física. }\end{array}$ & $\begin{array}{l}\text { ALFIERI. et } \\
\text { al., } 2016\end{array}$ & $\begin{array}{l}86 \\
\text { universitário } \\
\text { s. A partir de } \\
18 \text { anos }\end{array}$ & $\begin{array}{l}\text { Ambos } \\
\text { gêneros. }\end{array}$ & $\begin{array}{l}\text { Analisar a prevalência } \\
\text { da } \\
\text { dor lombar em } \\
\text { universitários e sua } \\
\text { relação com o estilo de } \\
\text { vida e o nível de } \\
\text { atividade física. }\end{array}$ & $\begin{array}{l}\text { Conclui-se que mais da } \\
\text { metade dos universitários da } \\
\text { área da saúde apresentam } \\
\text { lombalgia, e que há relação } \\
\text { entre estilo de vida. Apesar } \\
\text { disso, não houve relação entre } \\
\text { o nível de atividade física e a } \\
\text { presença de lombalgia. }\end{array}$ \\
\hline $\begin{array}{lcr}\text { Frequência } & \text { e } & \text { Fatores } \\
\text { associados a } & \text { dores } \\
\text { musculoesqueléticas } & \text { em } \\
\text { estudantes universitários. }\end{array}$ & $\begin{array}{l}\text { GOMES } \\
\text { NETO, M, et } \\
\text { al, 2016. }\end{array}$ & $\begin{array}{l}200 \\
\text { universitário } \\
\text { s. A partir de } \\
27 \text { anos }\end{array}$ & $\begin{array}{l}81 \% \text { eram } \\
\text { do sexo } \\
\text { feminino e } \\
19 \% \\
\text { masculino }\end{array}$ & $\begin{array}{l}\text { Estimar a frequência de } \\
\text { dores e desconforto, } \\
\text { identificando os } \\
\text { prováveis fatores de } \\
\text { risco percebidos pôr em } \\
\text { estudantes } \\
\text { universitários. }\end{array}$ & $\begin{array}{l}\text { A frequência de dores } \\
\text { musculoesqueléticas em } \\
\text { universitários foi alta, tendo } \\
\text { fatores relacionados tanto ao } \\
\text { ambiente ocupacional } \\
\text { acadêmico } \\
\text { desencadeamento no } \\
\text { potencialização deste agravo, } \\
\text { sendo necessária a elaboração } \\
\text { de ações preventivas desde os } \\
\text { cuidados ao tratamento dos } \\
\text { indivíduos que sofrem com } \\
\text { esses distúrbios. }\end{array}$ \\
\hline $\begin{array}{lrr}\text { Lombalgia, } & \text { hábitos } \\
\text { posturais e comportamentais } \\
\text { em acadêmicos } & \text { de } \\
\text { Fisioterapia e } & \\
\text { Psicologia de } & \text { uma } \\
\text { instituição de } & \text { ensino } \\
\text { superior } & & \end{array}$ & $\begin{array}{l}\text { SOUSA, P.O, } \\
\text { et al, } 2017 .\end{array}$ & $\begin{array}{l}171 \\
\text { universitário } \\
\text { s. Entre } 17 \text { e } \\
30 \text { anos. }\end{array}$ & $\begin{array}{l}66 \% \\
\text { feminino e } \\
34 \% \\
\text { masculino. }\end{array}$ & $\begin{array}{lr}\text { Analisar a prevalência e } \\
\text { associação } & \text { de } \\
\text { lombalgia, } & \text { hábitos } \\
\text { posturais } & \mathrm{e} \\
\text { comportamentais } & \mathrm{em} \\
\text { acadêmicos } & \mathrm{de} \\
\text { Fisioterapia } & \mathrm{e} \\
\text { Psicologia. } & \end{array}$ & $\begin{array}{l}\text { Houve elevada prevalência de } \\
\text { lombalgia relacionadas a } \\
\text { hábitos posturais } \\
\text { comportamentais } \\
\text { Inadequados, } \\
\text { significante a relação entre a } \\
\text { dor, prática, frequência e o } \\
\text { nível de competitividade da } \\
\text { atividade física, tempo e a } \\
\text { postura sentada para utilizar o } \\
\text { computador. }\end{array}$ \\
\hline
\end{tabular}




\begin{tabular}{|c|c|c|c|c|c|}
\hline $\begin{array}{l}\text { Prevalência de dor crônica e } \\
\text { fatores associados em } \\
\text { estudantes de medicina }\end{array}$ & $\begin{array}{l}\text { LOPES E } \\
\text { SILVA. et al., } \\
2017\end{array}$ & $\begin{array}{l}395 \\
\text { universitário } \\
\text { s. De } 21 \text { a } 25 \\
\text { Anos }\end{array}$ & $\begin{array}{l}\text { Feminino } \\
\text { com 64\% } \\
\text { Participante } \\
\text { s e } \\
\text { masculino } \\
\text { com } 36 \% \\
\text { participante } \\
\text { s }\end{array}$ & $\begin{array}{l}\text { Identificar a prevalência } \\
\text { de dor crônica em } \\
\text { estudantes de medicina } \\
\text { de uma Universidade do } \\
\text { Interior Paulista. }\end{array}$ & $\begin{array}{l}\text { A prevalência de dor crônica } \\
\text { com maior ocorrência foi no } \\
\text { sexo feminino. Em relação a } \\
\text { localização da dor, houve } \\
\text { maior prevalência na região } \\
\text { lombar e sacrococcígea. }\end{array}$ \\
\hline $\begin{array}{l}\text { Prevalência e fatores } \\
\text { associados a dor lombar em } \\
\text { estudantes de fisioterapia. }\end{array}$ & $\begin{array}{l}\text { MORAIS, } \\
\text { M.L, et al, } \\
2018 .\end{array}$ & $\begin{array}{l}410 \\
\text { universitário } \\
\text { s. A partir de } \\
18 \text { anos }\end{array}$ & $\begin{array}{l}72,9 \% \\
\text { feminino, } \\
27,1 \% \\
\text { masculino. }\end{array}$ & $\begin{array}{l}\text { Analisar os fatores } \\
\text { associados à dor } \\
\text { lombar em estudantes de } \\
\text { fisioterapia. }\end{array}$ & $\begin{array}{l}\text { Houve alta prevalência de dor } \\
\text { lombar nos universitários } \\
\text { pesquisados, porém mais } \\
\text { informações sobre a } \\
\text { prevalência de dor lombar } \\
\text { ainda se fazem necessárias } \\
\text { para compreender a dimensão } \\
\text { dos efeitos que essa dor } \\
\text { provoca, e encontrar meios } \\
\text { para uma melhor estratégia } \\
\text { preventiva e de intervenção. }\end{array}$ \\
\hline 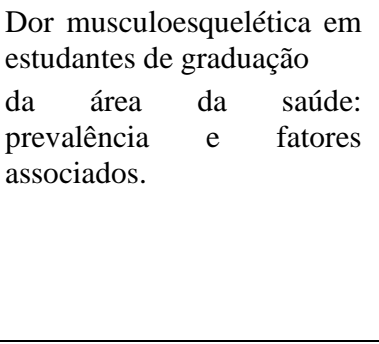 & $\begin{array}{l}\text { MORAIS, } \\
\text { B.X, et al, } \\
2019 \text {. }\end{array}$ & $\begin{array}{l}416 \\
\text { acadêmicos. } \\
\text { A partir de } \\
18 \text { anos }\end{array}$ & $\begin{array}{l}74,6 \% \\
\text { mulheres, } \\
25,4 \% \\
\text { masculino. }\end{array}$ & $\begin{array}{l}\text { verificar a prevalência e } \\
\text { os fatores associados à } \\
\text { dor musculoesquelética } \\
\text { em estudantes de } \\
\text { graduação da área da } \\
\text { saúde. }\end{array}$ & $\begin{array}{l}\text { Verificou-se um elevado percen } \\
\text { de dor musculoesquelética } \\
\text { estudantes de graduação da áre } \\
\text { saúde } 54,5 \% \text { em região lom } \\
\text { Visto que pode inter } \\
\text { negativamente na saúde e } \\
\text { no processo ensino- } \\
\begin{array}{l}\text { aprendizagem } \\
\text { população }\end{array}\end{array}$ \\
\hline $\begin{array}{l}\text { Diagnóstico de lombalgia } \\
\text { em universitários da área da } \\
\text { saúde em Tepic, Nayarit. }\end{array}$ & $\begin{array}{l}\text { CERVANTE } \\
\text { S - SOTO. et } \\
\text { al, } 2019 .\end{array}$ & $\begin{array}{l}90 \\
\text { universitário } \\
\text { s. A partir de } \\
18 \text { anos. }\end{array}$ & $\begin{array}{l}\text { Ambos os } \\
\text { gêneros. }\end{array}$ & $\begin{array}{l}\text { Determinar } \\
\text { prevalência de dor } \\
\text { lombar em estudantes } \\
\text { universitários de Tepic } \\
\text { Nayarit, México. }\end{array}$ & $\begin{array}{l}\text { As posturas foram um fator } \\
\text { determinante no } \\
\text { sofrimento de dores lombares. } \\
\text { alunos do Bacharelado em Nutr } \\
\text { e Fisioterapia estavam propens } \\
\text { sofrer e a maioria deles tinha } \\
\text { percepção dolorosa na re } \\
\text { lombar, devido às med } \\
\text { ergonômicas inadequadas } \\
\text { móveis da universidade. }\end{array}$ \\
\hline $\begin{array}{l}\text { Perfil da sintomatologia } \\
\text { dolorosa da coluna vertebral } \\
\text { em estudantes de graduação } \\
\text { em um município do } \\
\text { Maranhão }\end{array}$ & $\begin{array}{l}\text { DE SOUZA, } \\
\text { E.D.S, et al, } \\
2020 .\end{array}$ & $\begin{array}{l}46 \\
\text { universitário } \\
\text { s. }\end{array}$ & $\begin{array}{l}\text { Ambos os } \\
\text { gêneros. }\end{array}$ & 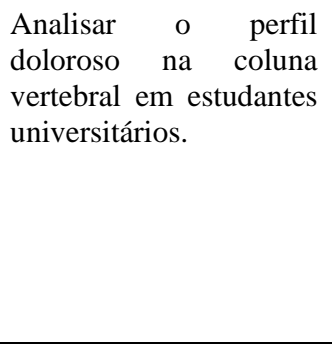 & $\begin{array}{l}\text { Foi identificando que a } \\
\text { maioria dos acadêmicos sentem } \\
\text { em repouso na coluna vertebral } \\
\text { predominância em região lom } \\
\text { isso provavelmente por conta } \\
\text { hábitos inadequados e por } \\
\text { praticarem exercício fí } \\
\text { diminuindo a qualidade de vida } \\
\text { mesmos }\end{array}$ \\
\hline $\begin{array}{l}\text { Avaliação do perfil de dor } \\
\text { lombar em estudantes de } \\
\text { fisioterapia. }\end{array}$ & $\begin{array}{l}\text { SILVA et al., } \\
2020\end{array}$ & $\begin{array}{l}186 \\
\text { universitário } \\
\text { s. A partir de } \\
18 \text { anos }\end{array}$ & $\begin{array}{l}89,6 \% \\
\text { mulheres, } \\
10,3 \% \\
\text { masculino. }\end{array}$ & $\begin{array}{l}\text { Avaliar o perfil de dor } \\
\text { lombar dos estudantes } \\
\text { de fisioterapia de uma } \\
\text { instituição superior de } \\
\text { ensino. }\end{array}$ & $\begin{array}{l}\text { Dos acadêmicos que respondera } \\
\text { questionário,61,7\% tiveram } \\
\text { pontuação elevada, demonstra } \\
\text { sentirem uma dor lon } \\
\text { significativa, afetando a qualid } \\
\text { de vida desses indivíduos e } \\
\text { consequência, sendo capaz } \\
\text { interferir no meio acadêmico } \\
\text { forma a diminuir seu desempe } \\
\text { A maioria são do sexo feminino }\end{array}$ \\
\hline $\begin{array}{l}\text { Dor e incapacidade na } \\
\text { região lombar referente a } \\
\text { lombalgia em alunos do } \\
\text { último período do curso de } \\
\text { fisioterapia em instituição de } \\
\text { ensino superior. }\end{array}$ & $\begin{array}{l}\text { CAMEY et } \\
\text { al., } 2020\end{array}$ & $\begin{array}{l}16 \\
\text { universitário } \\
\text { s. A partir de } \\
18 \text { anos }\end{array}$ & $\begin{array}{l}100 \% \\
\text { feminino, }\end{array}$ & $\begin{array}{l}\text { Quantificar } \\
\text { estatisticamente quadros } \\
\text { de lombalgia em alunos } \\
\text { de Fisioterapia dos } \\
\text { últimos períodos e } \\
\text { verificar a associação da } \\
\text { incapacidade com a dor } \\
\text { na região lombar em }\end{array}$ & $\begin{array}{l}\text { Concluímos que, houve presenç } \\
\text { lombalgia em } 50 \% \text { (8) da amo } \\
\text { (7) das alunas responderam } \\
\text { "não apresentam" e } 6 \% \text { (1) on } \\
\text { por não responder. Novas pesqu } \\
\text { na área devem ser realiza } \\
\text { buscando investigar o estilo de } \\
\text { e possíveis mecanismos }\end{array}$ \\
\hline
\end{tabular}




\begin{tabular}{|c|c|c|c|c|c|}
\hline & & & & $\begin{array}{l}\text { alunos do último } \\
\text { período do curso de } \\
\text { Fisioterapia em uma } \\
\text { instituição de ensino. }\end{array}$ & desenvolvimento da lombalgia. \\
\hline $\begin{array}{l}\text { Identificação do grau de } \\
\text { incapacidade física em } \\
\text { estudantes de uma } \\
\text { instituição de nível superior } \\
\text { do estado de alagoas com } \\
\text { dor lombar crônica. }\end{array}$ & $\begin{array}{l}\text { CARDOSO. } \\
\text { et al., } 2020\end{array}$ & $\begin{array}{l}300 \\
\text { universitário } \\
\text { s. A partir de } \\
18 \text { anos }\end{array}$ & $\begin{array}{l}90 \% \\
\text { mulheres e } \\
10 \% \\
\text { homens. }\end{array}$ & $\begin{array}{l}\text { Determinar o grau de } \\
\text { incapacidade física em } \\
\text { estudantes de nível } \\
\text { superior de uma } \\
\text { instituição particular do } \\
\text { estado de Alagoas com } \\
\text { dor lombar crônica. }\end{array}$ & $\begin{array}{l}\text { A predominância de } \\
\text { incapacidade física nos } \\
\text { estudantes com dor lombar } \\
\text { crônica foi leve. Mas grau } \\
\text { crônico ocupa uma parcela } \\
\text { significativa, portanto mesmo } \\
\text { com melhora dos episódios em } \\
\text { pouco tempo, alguns podem } \\
\text { evoluir para problemas } \\
\text { crônicos diminuindo a } \\
\text { qualidade de vida. }\end{array}$ \\
\hline $\begin{array}{l}\text { Prevalência e padrões de dor } \\
\text { musculoesquelética em } \\
\text { estudantes } \\
\text { de graduação em terapia } \\
\text { ocupacional e fisioterapia } \\
\text { em uma } \\
\text { universidade sul-africana }\end{array}$ & $\begin{array}{l}\text { OGUNLANA } \\
\text { O.M, et al., } \\
2021 .\end{array}$ & $\begin{array}{l}145 \\
\text { universitário } \\
\text { s. A partir de } \\
17 \text { anos }\end{array}$ & $\begin{array}{l}79,3 \% \\
\text { feminino, } \\
20,7 \% \\
\text { masculino. }\end{array}$ & $\begin{array}{l}\text { Investigar a prevalência, } \\
\text { o padrão e os possíveis } \\
\text { fatores de risco da dor } \\
\text { musculoesquelética } \\
\text { entre estudantes de } \\
\text { graduação em terapia } \\
\text { ocupacional } r \\
\text { fisioterapia em } \\
\text { universidade } \\
\text { africana. }\end{array}$ & $\begin{array}{l}\text { A dor musculoesquelética } \\
\text { altamente prevalente } \\
\text { estudantes de graduação } \\
\text { ciências da saúde, afetando } 64,4 \\
\text { região lombar. Esta } \\
\text { significativamente associada } \\
\text { posturas sedentárias e inadequ } \\
\text { na participação em ativid } \\
\text { físicas estruturadas. }\end{array}$ \\
\hline $\begin{array}{l}\text { Prevalência de sintomas } \\
\text { posturais } \\
\text { musculoesqueléticos entre } \\
\text { estudantes de odontologia } \\
\text { nos Emirados Árabes } \\
\text { Unidos }\end{array}$ & $\begin{array}{l}\text { Hashim. R., et } \\
\text { al., } 2021\end{array}$ & $\begin{array}{l}202 \\
\text { universitário } \\
\text { s }\end{array}$ & $\begin{array}{l}75,2 \% \\
\text { feminino, } \\
24,8 \% \\
\text { masculino }\end{array}$ & $\begin{array}{l}\text { Determinar } \\
\text { prevalência de dor no } \\
\text { pescoço, ombro e } \\
\text { lombar e examinar os } \\
\text { fatores associados à dor } \\
\text { musculoesquelética } \\
\text { (MSP) entre estudantes } \\
\text { de odontologia }\end{array}$ & $\begin{array}{l}\text { A prevalência de dores } \\
\text { musculoesqueléticas no } \\
\text { último ano é elevada entre os al } \\
\text { de graduação em odontolo } \\
\text { sendo mais comum em re } \\
\text { lombar, principalmente naqu } \\
\text { com história de } \\
\text { trauma, menos atividade } \\
\text { física, longas horas de sessões } \\
\text { clínicas e maior IMC. }\end{array}$ \\
\hline $\begin{array}{l}\text { Alteração postural, dor } \\
\text { lombar e a resistência dos } \\
\text { músculos estabilizadores do } \\
\text { tronco em jovens } \\
\text { universitários. }\end{array}$ & $\begin{array}{l}\text { FISCHER. } \\
2021\end{array}$ & $\begin{array}{l}40 \\
\text { universitária } \\
\text { s. A partir de } \\
20 \text { anos }\end{array}$ & $\begin{array}{l}100 \% \\
\text { Feminino. }\end{array}$ & $\begin{array}{l}\text { Identificar se existe } \\
\text { relação entre as } \\
\text { alterações posturais em } \\
\text { universitários com e sem } \\
\text { queixa de dor lombar e a } \\
\text { resistência dos músculos } \\
\text { estabilizadores do } \\
\text { tronco. }\end{array}$ & $\begin{array}{l}\text { Não existe relação direta } \\
\text { significativa entre as altera } \\
\text { posturais em jovens universita } \\
\text { com e sem queixa de dor lom } \\
\text { com a resistência dos músc } \\
\text { estabilizadores do tronco. }\end{array}$ \\
\hline
\end{tabular}

Fonte: Santos, Silva e Alencar (2021).

Esta revisão avaliou literaturas que reportam dados sobre a prevalência de lombalgia em universitários, com intuito de esclarecer a alta taxa de manifestação ocorrentes durante a graduação, onde foram estabelecidos pontos principais a serem abordados e discutidos no estudo.

Em seu estudo, Morais et al, (2019) observou prevalência de 54,5\% de dor lombar em estudantes de graduação na saúde. O mesmo autor evidenciou que existe relevância de dor musculoesquelética entre os estudantes no contexto acadêmico, visto que pode interferir negativamente na saúde e no processo ensino-aprendizagem dessa população.

Em outro estudo, Morais et al., (2018) a prevalência de dor lombar no último ano foi de 56,3\% nos estudantes afirmando que a execução das tarefas dos universitários mesmo que com pequenos movimentos ou a caminhada da faculdade para casa diminuem o hábito sedentário, assim contribuindo com um fator de proteção para dor lombar. Corroborando com o estudo anterior, Hashim et al., (2021) destacou por sua vez através de questionários alta prevalência de dores musculoesqueléticas no último ano de graduação em alunos de odontologia. 
Os resultados obtidos na pesquisa de Gomes neto et al., (2016) revelaram alta frequência de distúrbios osteomusculares percebidos entre os participantes, sendo (66\%) em região lombar, indicando uma frequência elevada de lombalgia entre os acadêmicos, o mesmo descreve que fatores existentes como sedentarismo, a permanência prolongada na posição sentada, à inadequação do mobiliário acadêmico e ao estilo de vida agitado que o aluno adota durante sua formação influenciam no acometimento dos sintomas de lombalgia .

De acordo com Alfieri et al., (2016) a prevalência de lombalgia foi identificada em 57\% dos 86 universitários abordados, reforçando assim o alto índice de dor entre estes estudantes. Mostrou que há relação da lombalgia e o estilo de vida, evidenciando assim que os hábitos de vida interferem nas queixas álgicas da coluna lombar. Apesar disso, porém não foram observadas diferenças em relação ao nível de atividade física e a presença de lombalgia.

No entanto no estudo de Souza et al. (2020) Correlaciona diferenças nos universitários participantes que não fizeram nenhuma atividade física e relataram dor com maior predominância foi a região lombar. $\mathrm{O}$ autor ressaltou que praticar exercícios físicos, com a ajuda de um profissional, é essencial para que os jovens tenham uma qualidade de vida excelente, desse modo, diminuindo suas dores musculoesqueléticas na coluna vertebral.

Outro autor que aborda o item qualidade de vida em seu estudo foi Cardoso et al., (2020) no qual ressalva que a predominância de incapacidade física nos estudantes com dor lombar crônica foi leve, ressaltando que mesmo que haja melhora dos episódios de dor lombar alguns destes podem evoluir para problemas crônicos, o que resultaria em alta despesa médica, redução de rendimento no trabalho, interferindo assim na da qualidade de vida dos universitários.

Confirmando achados de estudos em anos anteriores, Ogunlana et al., (2021) afirma que 64,4\% dos participantes relataram lombalgia, e confirma que os universitários apresentaram alta prevalência de dor lombar por não praticar atividade física com frequência. Além disso Handrakis et al., (2012) diz que, atividade física, força, resistência abdominal e postura associados ao tratamento da lombalgia pode ter um impacto significativo na prevenção e reabilitação dessa condição, confirmando assim achados anteriores quanto a relação de atividade física e dor lombar.

Fischer, (2021) sugeri não existir relação direta e significativa entre as alterações posturais em jovens universitários com e sem queixa de dor lombar. Entretanto Cervantes-Soto et al., (2019) afirma que as posturas são um fator determinante no sofrimento de dores lombares, relacionando este importante achado em seu estudo às medidas ergonômicas inadequadas dos móveis da universidade.

Em relação ao sexo, notou-se que as mulheres têm mais chances de desenvolverem lombalgia do que os homens. Sousa et al., (2017) Em seus resultados referentes à dor lombar apresentaram prevalência de 80,7\%, com percentuais de: 61,4\% feminino e 19,3\% masculino. Corroborando com Lopes e Silva et al., (2017) em que a maior prevalência de dor crônica apresentado por 64,38\% na região lombar com recorrência em mulheres. Seguido por Camey et al., (2020) 50\% de sua amostra composta por mulheres apresentou lombalgia. Silva. et al., (2020) justifica seu estudo devido a pequena adesão do público masculino à pesquisa e provável maioria do público feminino. Morais et al., (2019) por sua vez salienta que isso pode acontecer, em especial, pela diferença de massa muscular, massa óssea, articulações mais frágeis e estatura corporal, quando comparadas ao sexo masculino.

A lombalgia está relacionada a um problema de saúde pública, isto por conta de múltiplos fatores que associados ao cotidiano afetam grande parte da população. No meio acadêmico é possível observar que os universitários podem estar muito mais vulneráveis a tal acometimento pelo ambiente ao qual estão expostos, vivenciando grandes mudanças de rotina.

\section{Conclusão}

Com base no que foi apresentado é possível observar que houve alta prevalência de lombalgia em universitários, a maioria dos artigos expostos apresentaram em comum o acometimento de mais da metade dos participantes com queixas de dor lombar, sendo o gênero feminino mais afetado. 
Dessa maneira as evidências mostraram que os fatores mais comuns para o surgimento de lombalgia nos universitários se dão principalmente por sedentarismo, períodos prolongados na postura sentada, posturas inadequadas, inatividade física, mudanças no estilo de vida. A prática de exercícios físicos é ressaltada de forma importante para prevenção.

Sugere-se que sejam tomadas medidas para ampliar as evidências, como novas publicações que abordem a temática e assim promover impactos positivos com intuito de esclarecer e conscientizar os universitários acerca da dimensão dos efeitos que a lombalgia pode acarretar e consequentemente incentivar nas estratégias preventivas e de intervenção para melhorias da saúde e bemestar, favorecendo conhecimentos e contribuindo na literatura.

\section{Agradecimentos}

Agradecemos primeiramente à Deus por nos permitir essa realização e assim damos mais um passo importante em nossas vidas e carreira, a nossa orientadora Indiara de Alencar por desempenhar tal função com dedicação e maestria suas correções, paciência e apoio nos permitiram chegar até aqui guiando nosso aprendizado, aos nossos familiares por sonhar juntamente conosco e nos dar todo apoio necessário, seremos para sempre gratas.

\section{Referências}

Alfieri, F. M., de Oliveira, N. C., Santana, I. E. F. C., do Prado Ferreira, K. M., \& Pedro, R. D. M. (2016). Prevalência de dor lombar em universitários da saúde e sua relação com estilo de vida e nível de atividade física. $C E P, 5858,001$.

Barros, S. S. d., Ângelo, R. d. C. d. O., \& Uchôa, É. P. B. L. (2011). Lombalgia ocupacional e a postura sentada. Revista Dor, 12(3), 226-230. https://doi.org/10.1590/s1806-00132011000300006

Bréder, V. F. (2018). Atividade física e lombalgia. Fisioterapia Brasil, 6(2), 157. https://doi.org/10.33233/fb.v6i2.1980

Braccialli, L. M. P., \& Vilarta, R. (2000). Aspectos a serem considerados na elaboração de programas de prevenção e orientação de problemas posturais. Revista Paulista De Educação Física, 14(2), 159-171. https://doi.org/10.11606/issn.2594-5904.rpef.2000.138610

Camey, L. U., Da Silva, M. D. S., \& Cunha, R. G. (2020). Dor e incapacidade na região lombar referente a lombalgia em alunos dos últimos períodos do curso de fisioterapia em uma instituição de ensino superior. NBC-Periódico Científico do Núcleo de Biociências, 10(19).

Cardoso, P. D. S., Santos, I. O., \& Barbosa, F. T. (2020). Identificação do grau de incapacidade física de estudantes de uma instituição de nível superior do estado de alagoas com dor lombar crônica. Semana de Pesquisa do Centro Universitário Tiradentes-SEMPESq-Alagoas, (8).

Carvalho, M. E. C. C., Lima, L. C., de Lira Terceiro, C. A., Pinto, D. R. L., Silva, M. N., Cozer, G. A., \& Couceiro, T. C. d. M. (2017). Lombalgia na gestação. Brazilian Journal of Anesthesiology, 67(3), 266-270. https://doi.org/10.1016/j.bjan.2016.03.002

Cervantes-Soto, Araceli Janeth, García-Saaib, Adilene Rubí, Torres-Bonilla, Xenia Yahaira, Castellanos-Magdaleno, Gerardo, \& Mercado-Mercado, Gilberto. (2019). Diagnóstico de lumbalgia en estudiantes universitarios del área de salud en Tepic, Nayarit. Medicina Legal de Costa Rica, 36(1), 43-53. http://www.scielo.sa.cr/scielo.php?script=sci_arttext\&pid=S1409-00152019000100043\&lng=en\&tlng=es.

Casarin, S. T., Porto, A. R., Gabatz, R. I. B., Bonow, C. A., Ribeiro, J. P., \& Mota, M. S. (2020). Tipos de revisão de literatura: considerações das editoras do Journal of Nursing and Health/Types of literature review: considerations of the editors of the Journal of Nursing and Health. Journal of Nursing and Health, 10(5).

Da Silva Martins, M., \& Cassiano Longen, W. (2017). Atividade física comunitária: Efeitos sobre a funcionalidade na lombalgia crônica. Revista Brasileira em Promoção da Saúde, 30(4), 1-7. https://doi.org/10.5020/18061230.2017.6659

De Souza, E. d. S., Jardim, P. R. N., Sá, H. C. D., Silva, D. G., Victor, E. C., Ferreira, V. S., Reis, A. G., Mendes, N. d. O., E Sousa, C. L. M., \& Samineses, S. G. (2020). Perfil da sintomatologia dolorosa da coluna vertebral em estudantes de graduação em um município do Maranhão. Revista Eletrônica Acervo Científico, 16, Artigo e5201. https://doi.org/10.25248/reac.e5201.2020

Ebenbichler, G. R., E. Oddsson, L. I., Kollmitzer, J., \& Erim, Z. (2001). Sensory-motor control of the lower back: Implications for rehabilitation. Medicine and Science in Sports and Exercise, 33(11), 1889-1898. https://doi.org/10.1097/00005768-200111000-00014

Fischer, R. D. A. (2021). Alteração postural, dor lombar e a resistência dos músculos estabilizadores do tronco em jovens universitários [Trabalho de conclusão de curso, Universidade Estadual Paulista — UNESP]. https://repositorio.unesp.br/handle/11449/205128.

Gomes-Neto, M., Sampaio, G. S., \& Santos, P. S. (2016). Frequência e fatores associados a dores musculoesqueléticas em estudantes universitários. Revista Pesquisa em Fisioterapia, 6(1). https://doi.org/10.17267/2238-2704rpf.v6i1.790

Handrakis, J. P., Friel, K., Hoeffner, F., Akinkunle, O., Genova, V., Isakov, E., Mathew, J., \& Vitulli, F. (2012). Key characteristics of low back pain and disability in college-aged adults: A pilot study. Archives of Physical Medicine and Rehabilitation, 93(7), 1217-1224. https://doi.org/10.1016/j.apmr.2012.02.013

Hashim, R., Salah, A., Mayahi, F., \& Haidary, S. (2021). Prevalence of postural musculoskeletal symptoms among dental students in United Arab Emirates. BMC Musculoskeletal Disorders, 22(1). https://doi.org/10.1186/s12891-020-03887-x 
Leite, A. A. A. d. S., Santos, L. D. S., De Araújo, M. O., \& Cavalcante Neto, J. L. (2015). Dor lombar e exercício físico: Uma revisão sistemática. Revista Baiana de Saúde Pública, 39(2), 442. https://doi.org/10.22278/2318-2660.2015.v39.n2.a1709

Miranda, E. (2007). Coluna vertebral: anatomia, biomecânica, patologia, posturologia, testes neuromusculares, avaliação, exercícios complementares.

Morais, B. X., Dalmolin, G. d. L., Andolhe, R., Dullius, A. I. d. S., \& Rocha, L. P. (2019). Musculoskeletal pain in undergraduate health students: Prevalence and associated factors. Revista Da Escola De Enfermagem Da USP, 53. https://doi.org/10.1590/s1980-220x2018014403444

Morais, M. L., Silva, V. K. O., \& Silva, J. M. N. d. (2018). Prevalence of low back pain and associated factors among physiotherapy students. Brazilian Journal of Pain, 1(3). https://doi.org/10.5935/2595-0118.20180047

Ogunlana, M. O., Govender, P., \& Oyewole, O. O. (2021). Prevalence and patterns of musculoskeletal pain among undergraduate students of occupational therapy and physiotherapy in a South African university. Hong Kong Physiotherapy Journal, 41(01), 35-43. https://doi.org/10.1142/s1013702521500037

Ribeiro Campos, J., Barros Ferreira, J., \& Cavalcante Silva de Morais, K. (2018). A Kinesio Taping® no tratamento da dor em mulheres com lombalgia crônica. Revista InterScientia, 6(1), 42-54. Recuperado de https://periodicos.unipe.br/index.php/interscientia/article/view/597

Santos, J. J. A. d., Saracini, N., Silva, W. C. d., Guilherme, J. H., Costa, T. A. d., \& Gonçalves e Silva, M.-R. D. A. (2014). Estilo de vida relacionado à saúde de estudantes universitários: Comparação entre ingressantes e concluintes. ABCS Health Sciences, 39(1). https://doi.org/10.7322/abcshs.v39i1.256

Santos, V. R. M. (2011). Avaliação biomecânica dos ajustes posturais em indivíduos com dor lombar [PublishedVersion, Universidade de São Paulo]. http://www.teses.usp.br/teses/disponiveis/39/39132/tde-13012012-150444/

Serlachius, A., Hamer, M., \& Wardle, J. (2007). Stress and weight change in university students in the United Kingdom. Physiology \& Behavior, 92(4), 548-553. https://doi.org/10.1016/j.physbeh.2007.04.032

Silva, A. L. e., Smaidi, K., Pires, M. H. R., \& Pires, O. C. (2017). Prevalence of chronic pain and associated factors among medical students. Revista Dor, 18(2). https://doi.org/10.5935/1806-0013.20170022

Silva, G. T., Sardinha, I. S. N., Rossi, J. V., Angelim, L. S., De Resende, G. O., \& Nishi, M. (2020). Avaliação do perfil de dor lombar em estudante de fisioterapia. Anais da Mostra Acadêmica do Curso de Fisioterapia, 8(2), 1-7.

Sousa, P. O., Leal, S. S., \& De Carvalho, M. E. I. M. (2018). Lombalgia, hábitos posturais e comportamentais em acadêmicos de Fisioterapia e Psicologia de uma instituição de ensino superior. Fisioterapia Brasil, 18(5), 563. https://doi.org/10.33233/fb.v18i5.1551 